\title{
Colloidal Synthesis of Silver Nanoprisms in Aqueous Medium: Influence of Chemical Compounds in UV/Vis Absorption Spectra
}

\author{
Josivandro N. Silva ${ }^{1,2^{*}}$, Jamil Saade ${ }^{1,2}$, Patricia M. A. Farias ${ }^{1,2}$, Eduardo Henrique Lago Falcão ${ }^{2}$ \\ ${ }^{1}$ Research Group on Nanostructures and Biological Interfaces (NIB), Federal University of Pernambuco (UFPE), Recife, Brazil \\ ${ }^{2}$ Graduate Program on Materials Science (PGMTR), Federal University of Pernambuco (UFPE), Recife, Brazil \\ Email: ${ }^{*}$ josivandro.silva@ufpe.br
}

Received May 25, 2013; revised June 25, 2013; accepted July 3, 2013

Copyright (C) 2013 Josivandro N. Silva et al. This is an open access article distributed under the Creative Commons Attribution License, which permits unrestricted use, distribution, and reproduction in any medium, provided the original work is properly cited.

\begin{abstract}
In this work we explore the influence of the factors that affect the formation of silver nanoprisms (AgNPs) with distinct size and size distribution as well as the most intense absorption peak shift. Box-Behnken design analysis was applied to optimize the production of silver nanoprism via colloidal synthesis. The analysis of the responses was based on the nanoprisms plasmon peak wavelength related to in-plane dipole (tunable band) at $\lambda_{\max } \geq 420 \mathrm{~nm}$. The obtained results indicate that Silver ion is the main variable for the tuning of the size and the Localized Surface Plasmon Resonance (LSPR) frequency. By using the Box-Behnken design it was possible to synthesize nanoparticles with a predictable size and to establish a rigorous control of the plasmon frequency at the range of 600 up to $800 \mathrm{~nm}$.
\end{abstract}

Keywords: Silver Nanoprisms; Localized Surface Plasmon Resonance; Box-Behnken Design

\section{Introduction}

Practical and easy methodologies that enable the control of size and shape of metallic nanoparticles are quite of useful in nanotechnology, since several optical and electronic properties are strongly correlated to these characteristics [1]. In particular, silver nanoprisms (AgNPs) present very singular features related to the Localized Surface Plasmon Resonance (LSPR) effect, such as the intense bands that can be easily tunable and a significant contribution to the enhancement of the emissive process $[1,2]$.

Nowadays, silver nanoprisms have mostly been prepared by chemical methods or by assisted methods. Both methods depend on the control of some variables such as: metal precursor, stabilizers and reducing agents present in solution (reaction medium). Chemical methods use stabilizers (e.g. sodium citrate, SCT) and oxidizing agents (e.g. hydrogen peroxide, $\mathrm{H}_{2} \mathrm{O}_{2}$ ) to produce oxidative etching at the reaction medium. This occurs when a ligand and $\mathrm{O}_{2}$ are present. This combination may result in a powerful oxidation for both nuclei and seeds [3]. In such case, the seeds at the resulting colloid present sig-

"Corresponding author. nificant surface defects. These clusters will evolve to hexagonal or triangular plates [4]. Assisted methods use an external energy source like visible or ultraviolet light to reduce silver ions $\left(\mathrm{Ag}^{+}\right)$, do not make use of oxidizing agents and lead silver ions to grow in an anisotropic way, giving rise to the silver nanoparticles. Assisted methods tend to be more time consuming than chemical methods [5]. Both methods produce tipped and well formed AgNPs using low $\mathrm{Ag}^{+}$concentrations such as $0.1 \mathrm{mmol} \cdot \mathrm{L}^{-1}$ [4-6]. Assisted methods produce AgNPs that absorb between $500-1400 \mathrm{~nm}$ [5]. Chemical methods have produced AgNPs with tunable peak around of $800 \mathrm{~nm}[6,7]$. Nanoparticles with large absorption wavelength (around $1000 \mathrm{~nm}$ ) could be used, for instance, to coat optical fibers, reducing losses and thus improving signal quality [5].

As far as we know, there is a lack both of systematic studies on the synthesis parameters that enable the control of the nanoparticles' dimensions and of investigations about the contribution of each component over the size dispersion of the resulting particles of colloidal system. In this work the effect of the interaction between the components $\mathrm{Ag}^{+}, \mathrm{H}_{2} \mathrm{O}_{2}$, SCT and $\mathrm{NaBH}_{4}$ on the formation of colloidal AgNPs via chemical reduction method is investigated. We also discuss the role of the oxidizing 
agent $\mathrm{H}_{2} \mathrm{O}_{2}$ on the formation of stacking faults of the seed colloids, without which the anisotropy required for the rise of prismatic nanoparticles does not occur. The Box-Behnken [8] method for the Design of Experiments (DOE) was used absorption spectrum of the AgNPs which present three LSPR bands at distinct wavelengths.

\section{Materials and Methods}

\subsection{Materials}

All reactants (analytical grade) were purchased from Aldrich. Silver nitrate $\left(\mathrm{AgNO}_{3}, \geq 99 \%\right)$ was used as metallic precursor. Tribasic sodium citrate $\left(\mathrm{Na}_{3} \mathrm{C}_{6} \mathrm{H}_{5} \mathrm{O}_{7}, \geq 99 \%\right)$ was used as stabilizing agent. Sodium borohidrate $(\geq 98 \%)$ was used as reducing agent and hydrogen peroxide $\left(\mathrm{H}_{2} \mathrm{O}_{2}\right.$, $30 \mathrm{wt} \%$ ) was used as oxidizing agent. Milli-Q deionized water (conductivity about $18 \mathrm{M} \Omega$ ) was used to prepare the aqueous solution.

\subsection{Synthesis of Silver Nanoprisms}

The colloidal synthesis method proposed by Shi and coworkers [6] was modified in order to achieve control over the absorption wavelength of the colloidal AgNPs. Typically, $30 \mathrm{~mL}$ of Milli-Q deionized water and $1.5 \mathrm{~mL}$ of sodium citrate (SCT) $30 \mathrm{mmol} \cdot \mathrm{L}^{-1}$ were added into a $100 \mathrm{~mL}$ beaker under vigorous stirring. The amounts of the chemicals reagents added were modified as follows: $\mathrm{AgNO}_{3}$ level $30 \mathrm{mmol} \cdot \mathrm{L}^{-1}$ (30 and $70 \mu \mathrm{L}$ ), $\mathrm{H}_{2} \mathrm{O}_{2} 30 \%$ (40 and $60 \mu \mathrm{L}$ ) and $\mathrm{NaBH}_{4} 100 \mathrm{mmol} \cdot \mathrm{L}^{-1}(150$ and $200 \mu \mathrm{L})$.

\subsection{Box-Behnken Design}

Response Surface Methodology (RSM) was employed for experimental design, data analysis and model building, as well as the software packages Design Expert and Statistica 8. A Box-Behnken design with three variables was used to establish the model [8]: $\mathrm{AgNO}_{3}$ level $\left(x_{1}\right)$, $\mathrm{H}_{2} \mathrm{O}_{2}\left(x_{2}\right)$ and $\mathrm{NaBH}_{4}\left(x_{3}\right)$, with three levels for each variable. The dependent variable was the main band with variable wavelength. Symbols and levels are shown in Table 1. Five replicates at the central point of the designed model were used to estimate the pure error sum of squares.

\subsection{Analysis}

All colloids obtained by Box-Behnken design were characterized by UV-Vis Spectroscopy (Ocean Optics USB4000-UV-VIS spectrophotometer). The morphology of the samples was analyzed by Transmission Electronic Microscopy (TEM, FEI Morgagni 268D operating at 100 $\mathrm{kV})$. The samples were diluted in aqueous medium and dropped into a carbon grid. The images were analyzed with the software Image-Pro Plus 6, in order to quantify particle size and size distribution.
Table 1. Box-Behnken design and the response wavelength for $\mathrm{AgNO}_{3}$ formation.

\begin{tabular}{cccccccc}
\hline Levels & \multicolumn{3}{c}{ Numeric } & \multicolumn{3}{c}{ Coded } & Response \\
\hline Experiments & $\boldsymbol{x}_{1}$ & $\boldsymbol{x}_{\mathbf{2}}$ & $\boldsymbol{x}_{3}$ & $\boldsymbol{x}_{\mathbf{1}}$ & $\boldsymbol{x}_{\mathbf{2}}$ & $\boldsymbol{x}_{3}$ & $\boldsymbol{\lambda}(\mathbf{n m})$ \\
\hline 1 & 30 & 40 & 175 & - & - & 0 & 67,612 \\
2 & 70 & 40 & 175 & + & - & 0 & 48,491 \\
3 & 30 & 60 & 175 & - & + & 0 & 66,205 \\
4 & 70 & 60 & 175 & + & + & 0 & 52,488 \\
5 & 30 & 50 & 150 & - & 0 & - & 95,151 \\
6 & 70 & 50 & 150 & + & 0 & - & 47,627 \\
7 & 30 & 50 & 200 & - & 0 & + & 80,056 \\
8 & 70 & 50 & 200 & + & 0 & + & 52,436 \\
9 & 50 & 40 & 150 & 0 & - & - & 56,232 \\
10 & 50 & 60 & 150 & 0 & + & - & 64,334 \\
11 & 50 & 40 & 200 & 0 & - & + & 58,821 \\
12 & 50 & 60 & 200 & 0 & + & + & 60,938 \\
13 & 50 & 50 & 175 & 0 & 0 & 0 & 58,097 \\
14 & 50 & 50 & 175 & 0 & 0 & 0 & 58,899 \\
15 & 50 & 50 & 175 & 0 & 0 & 0 & 60,113 \\
16 & 50 & 50 & 175 & 0 & 0 & 0 & 59,010 \\
17 & 50 & 50 & 175 & 0 & 0 & 0 & 59,512 \\
\hline & & & & & & &
\end{tabular}

\section{Results and Discussion}

\section{1. $\mathrm{H}_{2} \mathrm{O}_{2}$ and the Formation of $\mathrm{Ag}$ Nanoprisms}

Formation of the AgNPs strongly depends on the chemical characteristics of the stabilizer and oxidizing agent (functional groups, strength of the oxidant agent, etc.). On the synthesis reported in this work, SCT was used as stabilizer. Other polymers containing $\mathrm{OH}$ groups also can be used, producing the same effect as SCT [9]. However, the presence of either the oxidant or stabilizer is not enough to form the AgNPs. In order to ensure AgNP formation, a mixing of these two components is needed. They must be both present at the solution, to promote the formation of an oxidative etching into the solution $[3,4]$.

To ensure AgNP formation, both components must be present, so that an oxidative etching is promoted. In the work reported here, the $\mathrm{H}_{2} \mathrm{O}_{2}$ concentration was kept between $0.4 \%$ and $0.6 \%(\mathrm{~V} / \mathrm{V})$, although concentrations as high as $1 \%$ still produced AgNPs. Higher concentrations of the oxidant generally resulted in spherical particles, rather than the desired nanoprisms. The solution with acid $\mathrm{pH}$ looses color, indicating the particles' demineralization. UV-Vis spectra confirms that the absence of $\mathrm{H}_{2} \mathrm{O}_{2}$ also results in the formation of spherical particles instead of nanoprisms. Mixing $\mathrm{H}_{2} \mathrm{O}_{2}$ to SCT favors the appearance of stacking faults in the seed colloid 
through an oxidative process early on, prior to the addition of $\mathrm{NaBH}_{4}$. The equations below illustrate the reactions that $\mathrm{H}_{2} \mathrm{O}_{2}$ undergoes on the silver cluster surface (Figure 1).

The peroxide anion $\left(\mathrm{HOO}^{-}\right)$is quite unstable and easily decomposes into the hydroxyl radical $\left(\mathrm{HO}^{-}\right)$, which is highly reactive and acts as a very strong oxidizing agent.

In addition to the Box-Behnken design, other experiments varying the $\mathrm{H}_{2} \mathrm{O}_{2}$ concentration were performed. It was observed that for volumes higher than $60 \mu \mathrm{L}, \mathrm{pH}$ value increases to beyond 8.5 and the newly formed AgNPs dissolve rapidly. This occurs because prismatic particles can have specific facets, such as $\{111\}$, more exposed than other morphologies (such as spheres and rods). Thus, under such conditions, prismatic nanoparticles are much more reactive towards the oxygen present in solution than other particle shapes $[4,10]$. This was observed during the experiments we've performed, and preferentially occurs at sites that present a small curvature radius (sharp edges and corners) [11]. Furthermore, other studies have also provided evidence that polycrystalline AgNPs will dissolve more quickly than singlecrystalline AgNPs [3,11,12]. The proposed explanation for this behavior is that polycrystalline particles contain high-energy defects at grain boundaries, which provide active sites for oxidation and dissolution [13].

\subsection{Spectroscopic and Morphologic Characteristics of Silver Nanoprisms}

In a typical UV-Vis spectrum of AgNPs, three absorption peaks can be found (Figure 2). The weak, narrow peak at $330 \mathrm{~nm}$ is assigned to out of plane dipole that corresponds to oscillations of the metal surface electrons along the nanoparticle thickness. There are two more bands with low intensities $(400-550 \mathrm{~nm})$ that are assigned to in plane quadrupole. The most intense peak is assigned to in plane dipole and its maximum is very sensitive to synthesis parameters, ranging between 500 to $950 \mathrm{~nm}$.

All UV-Vis spectra present three peaks, but only samples 1, 5, 7, 8, 9 correspond to well formed AgNPs. Figure 3 shows representative TEM images of four colloid samples (5, 7, 8 and 9) exhibiting different size distributions with edges and tips well formed.

According to UV-Vis spectra (Figure 2), the AgNPs formed present distinct size, since the position of the main band is related to the area of the formed particles. A linear correlation was observed for size distribution/po-

$$
\begin{aligned}
& \mathrm{H}_{2} \mathrm{O}_{2} \longrightarrow \mathrm{H}^{+}+\mathrm{HOO}^{-}\left(\mathrm{HOO}^{-}\right) \longrightarrow \mathrm{O}+\mathrm{OH}^{-} \\
& \mathrm{H}_{2} \mathrm{O}_{2}+\mathrm{HOO}^{-} \longrightarrow \mathrm{HO}+\mathrm{O}_{2}^{-}+\mathrm{H}_{2} \mathrm{O}
\end{aligned}
$$

Figure 1. Mechanism of interaction of hydrogen peroxide with the surface of the silver nanoparticles.

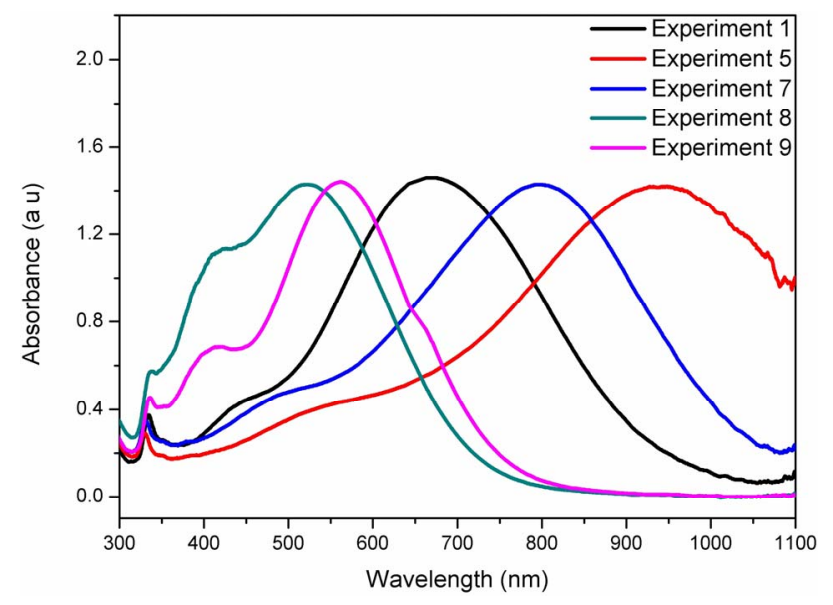

Figure 2. Normalized UV-Vis spectra for five samples from Box-Behnken design. Experiments curves 7, 8 and 9 were multiplied by a factor 1.2.

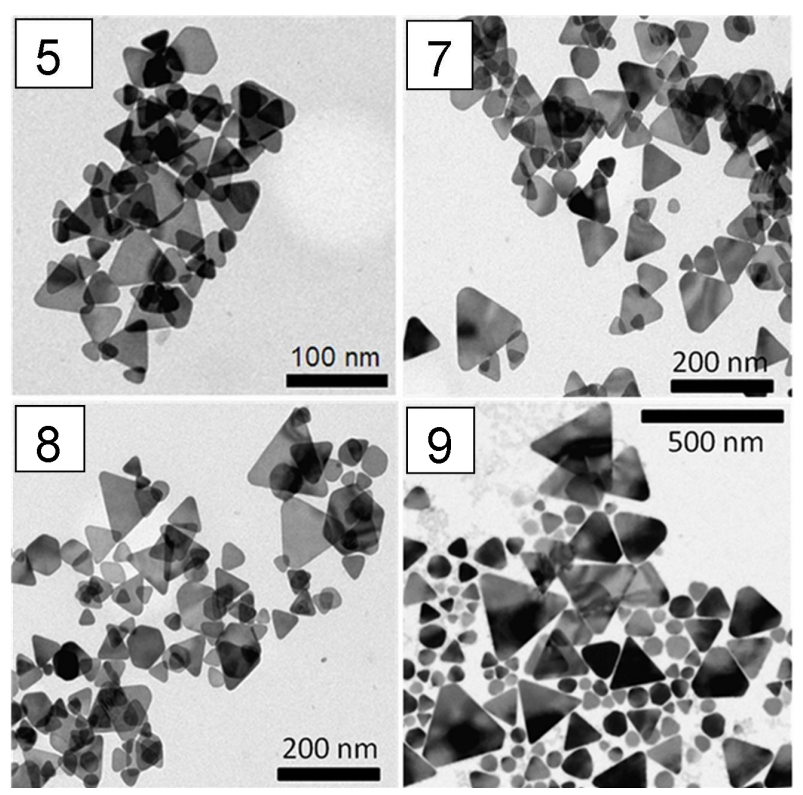

Figure 3. TEM images for experiments 5, 7, 8 and 9 realized on Box-Behnken Design.

sition of main peak maximum for experiments 5, 7, 8 and 9 , whose values were $58 \pm 10.15 \mathrm{~nm}, 73 \pm 15 \mathrm{~nm}, 96 \pm$ $21 \mathrm{~nm}$ and $225 \pm 44.63 \mathrm{~nm}$, respectively. Spherical and nanoplate particles of different sizes were also observed. This indicates that the silver ions, in the concentrations used in these experiments, did not convert all silver seed colloid into AgNPs. Hence, it was necessary to carry out distinct centrifugation steps in order to separate the different particle sizes present in the solution.

\subsection{Effect of $\mathrm{AgNO}_{3}, \mathrm{H}_{2} \mathrm{O}_{2}$, and $\mathrm{NaBH}_{4}$ Concentrations on AgNP Formation}

The three-dimensional response surface plots shown in Figures 4-6 evidence the effects of $\mathrm{AgNO}_{3}, \mathrm{H}_{2} \mathrm{O}_{2}$, and 


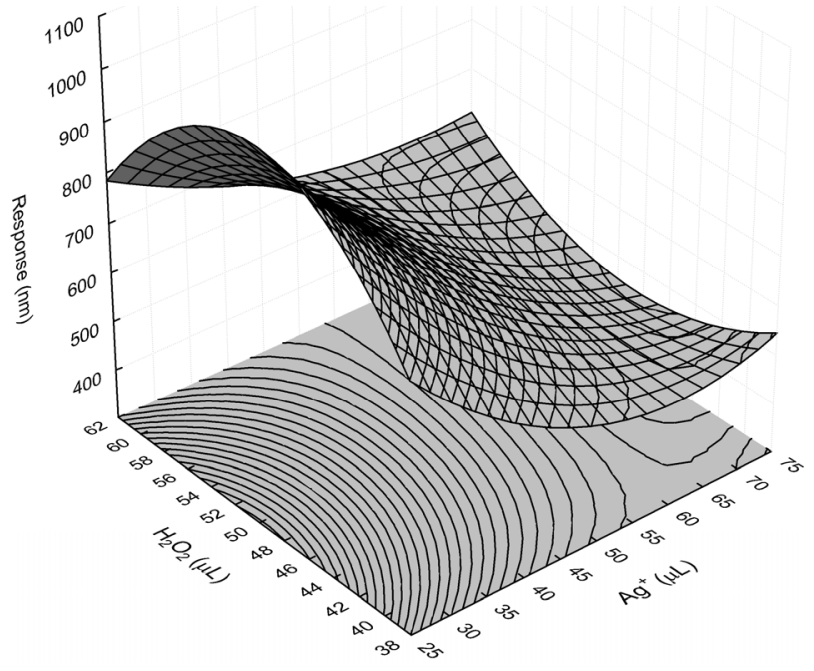

Figure 4. Response surface plot showing the effects of $\mathrm{Ag}^{+}$ level and $\mathrm{H}_{2} \mathrm{O}_{2}$ on the formation of AgNPs. $\mathrm{NaBH}_{4}$ remained constant at $150 \mu \mathrm{L}$.

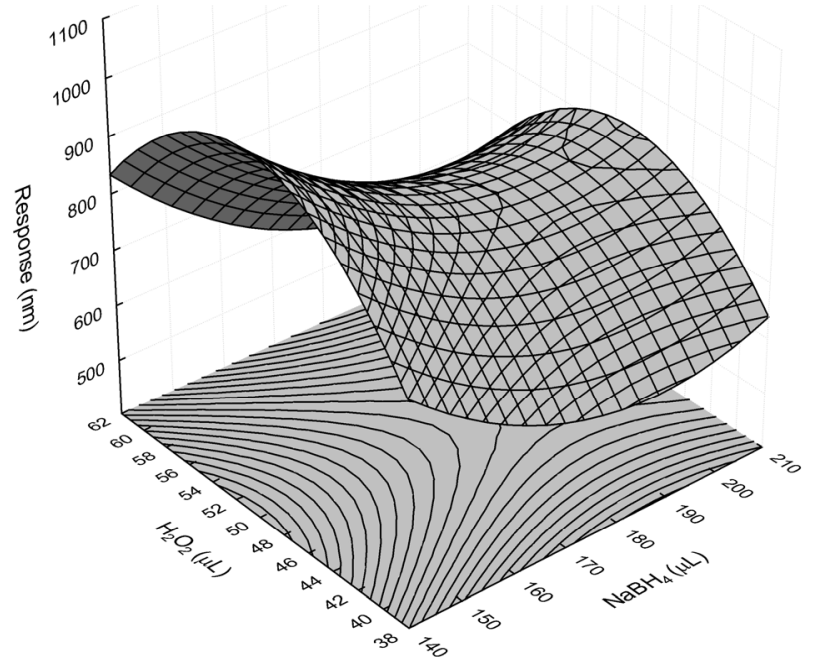

Figure 5. Response surface plot showing the effects of $\mathrm{NaBH}_{4}$ level and $\mathrm{H}_{2} \mathrm{O}_{2}$ on the formation of $\mathrm{AgNPs}$. $\mathrm{Ag}^{+}$remained constant at $30 \mu \mathrm{L}$.

$\mathrm{NaBH}_{4}$ levels, and their interactions on the formation of AgNPs. From the results obtained, it was observed that each factor played an apparent role in the preparation of AgNPs. As shown in Figure 4 the maximum shift of the tunable band of AgNPs increased gradually when the $\mathrm{H}_{2} \mathrm{O}_{2}$ level is kept between $40-60 \mu \mathrm{L}$, with a low level of $\mathrm{Ag}^{+}$. When the $\mathrm{Ag}^{+}$level and $\mathrm{H}_{2} \mathrm{O}_{2}$ were kept constant within the range under investigation, the shift module of the tunable band of the AgNPs formed increased exhibiting a dependence with the $\mathrm{NaBH}_{4}$ volume added (Figures 4 and 5 ).

\subsection{Model Fitting and Optimization}

The mathematical model representing the principal wave-

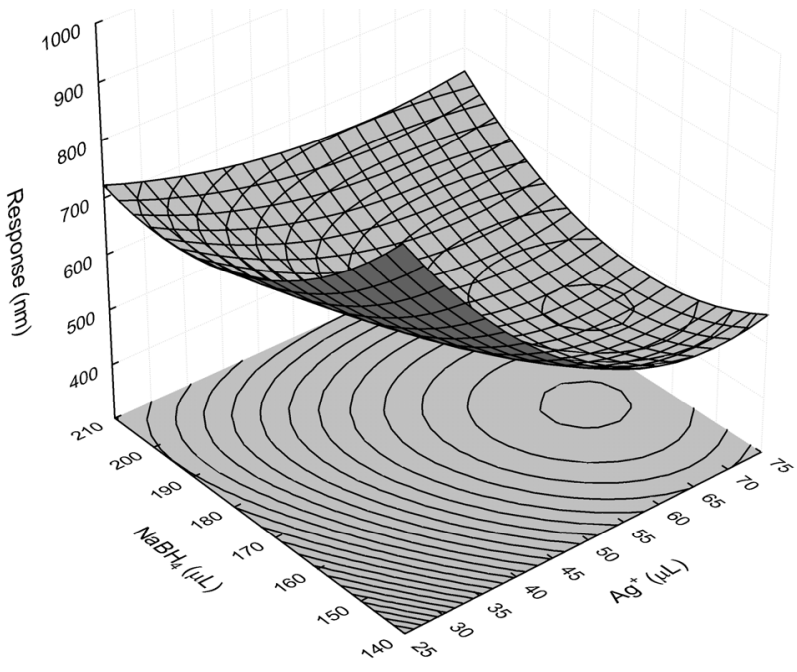

Figure 6. Response surface plot showing the effects of $\mathrm{Ag}^{+}$ level and $\mathrm{NaBH}_{4}$ on the formation of AgNPs. $\mathrm{H}_{2} \mathrm{O}_{2}$ remained constant at $40 \mu \mathrm{L}$.

lenght shift for AgNPs as a function of the independent variables in the region under investigation was expressed by the following equation:

$$
\begin{aligned}
y= & 591.26-134.98 * x_{1}+16.01 * x_{2}-13.51 * x_{1} * x_{2} \\
& +49.76 * x_{1} * x_{3}-14.96 * x_{2} * x_{3}+41.55 * x_{1}^{2} \\
& -45.82 * x_{2}^{2}+55.37 * x_{3}^{2}
\end{aligned}
$$

where $y$ represents the shift of principal wavelength, and $x_{1}, x_{2}$ and $x_{3}$ are the coded variables for $\mathrm{AgNO}_{3}, \mathrm{H}_{2} \mathrm{O}_{2}$ and $\mathrm{NaBH}_{4}$ levels, respectively.

Exploration and optimization of a fitted model may produce misleading results unless the model exhibits a good fitting. Thus, checking the model adequacy is essential. The model P-value of the AgNPs synthesis was 0.0137 (Table 2), indicating that the model fitting was significant. However, the "lack of fit" value was 0.0002 which means that this term was significant.

The coefficient $\left(\mathrm{R}^{2}\right)$ of determination is another important index for the measurement of the fitting degree. The small value of $\mathrm{R}^{2}$ indicates the poor relevance of the dependent variables in the model. The model can be well fitted with the actual data when $\mathrm{R}^{2}$ is near the unity. By analysis of variance, the $\mathrm{R}^{2}$ value of the model was determined to be 0.8855 , showing that the regression model emulated well the true behavior of the system.

According to this model three points with predicted wavelength were chosen to test the obtained results (Table 3).

Nanoparticles with LSPR bands into the visible region can be quite suitable to enhance the quantum efficiency of luminescent materials such as dyes and Quantum dots [14]. The plasmon bands of the metal particles can enhance the density of the electromagnetic field near their 
Table 2. Model fit analysis for the AgNPs synthesis.

\begin{tabular}{ccccc}
\hline Source & Degree of freedom & Sum of squares & Mean square & P-value \\
\hline Model & 9 & 189,000 & 21005.32 & 3492.55 \\
Residual & 7 & 24447.83 & 8.0137 \\
Lack of fit & 3 & 24223.13 & 0.0002 \\
Pure error & 4 & 224.70 & 56.17 \\
Total & 16 & 213,500 & \\
\hline
\end{tabular}

Table 3. Theoretical and experimental data for three experiment realized to obtain silver nanoprisms at predicted wavelengh values.

\begin{tabular}{cccccc}
\hline Run & $x_{1}(\mu \mathrm{L})$ & $x_{2}(\mu \mathrm{L})$ & $x_{3}(\mu \mathrm{L})$ & $\lambda_{\text {theo. }}(\mathrm{nm})$ & $\lambda_{\text {exp. }}(\mathrm{nm})$ \\
\hline 1 & 50 & 55 & 192 & $600 \pm 65.88$ & $615 \pm 31$ \\
2 & 35 & 56 & 200 & $700 \pm 75.70$ & $788 \pm 25$ \\
3 & 35 & 50 & 153 & $800 \pm 70.13$ & $752 \pm 42$ \\
\hline
\end{tabular}

neighbors, contributing to the enhancement of the local excitation of these luminescent structures. These kinds of materials are largely used for biological applications, such as tissue and cells markers for diagnosis and other studies [14].

\section{Conclusion}

Box-Behnken based experiments showed that it is possible to prepare silver nanoprisms with tunable wavelength. This indicated a distinct size distribution for each sample of silver nanoprisms synthesized. The interactions among all chemical components $\left(x_{1}, x_{2}\right.$, and $\left.x_{3}\right)$ used in the experiments were meaningful according to Table 2, however, the $x_{1}$ factor was the most important one. Statistic analysis ANOVA of the model was significant and indicated a good fitness with the data obtained. In order with fit of model were chosen three points with wavelength predicted values. All results presented a good approximation with predicted values.

\section{Acknowledgements}

This work was supported by Brazilian agencies CNPq and CAPES, as well as INFo (Instituto de Fotônica). The authors are grateful to CETENE for the use of Transmission Electronic Microscope.

\section{REFERENCES}

[1] K. L. Kelly, Z. E. Coronado, L. L. Zhao and G. C. Schatz, "The Optical Properties of Metal Nanoparticles: The Influence of Size, Shape, and Dielectric Environment," The Journal of Physical Chemistry B, Vol. 107, No. 3, 2003, pp. 668-677. doi:10.1021/jp026731y

[2] M. Grzelczak, J. Pérez-Juste, P. Mulvaney and L. M. Liz-Marzan, "Shape Control in Gold Nanoparticle Synthesis," Chemical Society Reviews, Vol. 37, No. 9, 2008, pp. 1783-1791.

[3] Y. Xia, Y. Xiong, B. Lim and S. Skrabalak, "Shape-Controlled Synthesis of Metal Nanocrystals: Simple Chemistry Meets Complex Physics?" Angewandte Chemie International Edition, Vol. 48, No. 1, 2009, pp. 60-103. doi:10.1002/anie. 200802248

[4] B. Wiley, T. Herricks, Y. Sun and Y. Xia, "Polyol Synthesis of Silver Nanoparticles: Use of Chloride and Oxygen to Promote the Formation of Single-Crystal, Truncated Cubes and Tetrahedrons," Nano Letter, Vol. 4, No. 9, 2004, pp. 1733-1739. doi:10.1021/n1048912c

[5] V. Bastys, I. Pastoriza-Santos, B. Rodríguez-González, R. Vaisnoras and L. M. Liz-Marzán, "Formation of Silver Nanoprisms with Surface Plasmons at Communication Wavelengths," Advanced Functional Materials, Vol. 16, No. 6, 2006, pp. 766-773. doi:10.1002/adfm.200500667

[6] W. Shi and Z. Ma, "Amperometric Glucose Biosensor Based on a Triangular Silver Nanoprisms/Chitosan Composite Film as Immobilization Matrix," Biosensors and Bioelectronics, Vol. 26, No. 3, 2010, pp. 1098-1103. doi:10.1016/j.bios.2010.08.072

[7] G. Si, W. Shi, K. Li and Z. Ma, "Synthesis of PSSCapped Triangular Silver Nanoplates with Tunble SPR," Colloids and Surfaces A: Physicochemical and Engineering Aspects, Vol. 380, No. 1-3, 2011, pp. 257-260. doi:10.1016/j.colsurfa.2011.02.023

[8] G. E. P. Box, W. G. Hunter and J. S. Hunter, "Statistics for Experimenters-An Introduction to Design, Data Analysis and Model Building," Wiley, New York, 1978.

[9] W. Zhang, Y. Yao, N. Sullivan and Y. S. Chen, "Modeling the Primary Size Effects of Citrate-Coated Silver Nanoparticles on Their Ion Release Kinetics," Environmental Science \& Technology, Vol. 45, No. 10, 2011, pp. 4422-4428. doi:10.1021/es104205a

[10] J. Yang, Q. Zhang, J. Y. Lee and H. Too, "DissolutionRecrystallization Mechanism for the Conversion of Silver Nanospheres to Triangular Nanoplates," Journal of Colloid and Interface Science, Vol. 308, No. 1, 2007, pp. 157-161. doi:10.1016/j.jcis.2006.12.081

[11] J. L. Elechiguerra, L. Larios-Lopez, C. Liu, D. GarciaGutierrez, A. Camacho-Bragado and M. J. Yacaman, "Corrosion at the Nanoscale: The Case of Silver Nanowires and Nanoparticles," Chemistry of Materials, Vol. 17, No. 24, 2005, pp. 6042-6052. doi: $10.1021 / \mathrm{cm} 051532 \mathrm{n}$

[12] E. Petryayeva and U. J. Krull, "Localized Surface Plasmon Resonance: Nanostructures, Bioassays and Biosensing-A Review," Analitica Chimica Acta, Vol. 706, No. 1, 2011, pp. 8-24. doi:10.1016/j.aca.2011.08.020

[13] M. Tsuji, S. Gomi, Y. Maeda, M. Matsunaga, S. Hikino, 
K. Uto, T. Tsuji and H. Kawazumi, "Rapid Transformation from Spherical Nanoparticles, Nanorods, Cubes, or Bipyramids to Triangular Prisms of Silver with PVP, Citrate, and $\mathrm{H}_{2} \mathrm{O}_{2}$," Langmuir, Vol. 28, No. 24, 2012, pp. 8845-8861. doi:10.1021/la3001027
[14] C. Loo, A. Lowery, N. Halas, J. West and R. Drezek, "Immunotargeted Nanoshells for Integrated Cancer Imaging and Therapy," Nano Letter, Vol. 5, No. 4, 2005, pp. 709-711. doi: $10.1021 / \mathrm{n} 1050127 \mathrm{~s}$ 